\title{
Ultrasound sonication with microbubbles disrupts blood vessels and enhances tumor treatments of anticancer nanodrug
}

This article was published in the following Dove Press journal:

International Journal of Nanomedicine

2I April 2012

Number of times this article has been viewed

\author{
Chung-Yin Lin ${ }^{*}$ \\ Hsiao-Ching Tseng ${ }^{*}$ \\ Heng-Ruei Shiu' \\ Ming-Fang $\mathrm{Wu}^{2}$ \\ Cheng-Ying $\mathrm{Chou}^{3}$ \\ Win-Li Lin ${ }^{1,4}$ \\ 'Institute of Biomedical Engineering, \\ ${ }^{2}$ Laboratory Animal Center, \\ ${ }^{3}$ Department of Bio-Industrial \\ Mechatronics Engineering, National \\ Taiwan University, Taipei, Taiwan; \\ ${ }^{4}$ Division of Medical Engineering \\ Research, National Health Research \\ Institutes, Miaoli, Taiwan \\ *These authors contributed equally \\ to this work
}

\begin{abstract}
Ultrasound (US) sonication with microbubbles (MBs) has the potential to disrupt blood vessels and enhance the delivery of drugs into the sonicated tissues. In this study, mouse ear tumors were employed to investigate the therapeutic effects of US, MBs, and pegylated liposomal doxorubicin (PLD) on tumors. Tumors started to receive treatments when they grew up to about $15 \mathrm{~mm}^{3}$ (early stage) with injection of PLD $10 \mathrm{mg} / \mathrm{kg}$, or up to $50 \mathrm{~mm}^{3}$ (medium stage) with PLD 6 (or 4) mg/kg. Experiments included the control, PLD alone, PLD + MBs + US, US alone, and MBs + US groups. The procedure for the PLD + MBs + US group was that PLD was injected first, MB (SonoVue) injection followed, and then US was immediately sonicated on the tumor. The results showed that: (1) US sonication with MBs was always able to produce a further hindrance to tumor growth for both early and medium-stage tumors; (2) for the medium-stage tumors, $6 \mathrm{mg} / \mathrm{kg}$ PLD alone was able to inhibit their growth, while it did not work for $4 \mathrm{mg} / \mathrm{kg}$ PLD alone; (3) with the application of MBs + US, $4 \mathrm{mg} / \mathrm{kg}$ PLD was able to inhibit the growth of medium-stage tumors; (4) for early stage tumors after the first treatment with a high dose of PLD alone $(10 \mathrm{mg} / \mathrm{kg})$, the tumor size still increased for several days and then decreased (a biphasic pattern); (5) MBs + US alone was able to hinder the growth of early stage tumors, but unable to hinder that of medium stage tumors. The results of histological examinations and blood perfusion measurements indicated that the application of MBs + US disrupts the tumor blood vessels and enhances the delivery of PLD into tumors to significantly inhibit tumor growth.
\end{abstract}

Keywords: ultrasound, microbubbles, nanodrug, mouse tumor, vascular disruption, tumor growth response

\section{Introduction}

The aim of chemotherapy is to deliver anticancer drug(s) to the targeted tumors with sufficiently high doses and to produce minimum side effects on normal tissue. ${ }^{1}$ Drug delivery with nanotechnology can result in preferential transport of drugs to tumors, owing to the enhanced permeability and retention effect. ${ }^{2}$ Vasculature plays a crucial role in tumor growth, metastasis, and drug delivery. The therapeutic strategy of nanodrugs is based on tumor microenvironment and on morphological and functional characteristics of tumor vasculature. ${ }^{3,4}$ Tumors commonly secrete vascular endothelial growth factor, and their vessels usually have wider intercellular junctions than normal vessels do, and many tumor vessels have fenestrated structures. ${ }^{5-7}$ The abnormal organization and structure of the tumor vasculature results in tortuous and leaky vessels and rather heterogeneous blood flow. ${ }^{8}$ Tumor vessels with different morphological characteristics coexist in the same tumor, and a previous study ${ }^{9}$ showed that the process of tumor growth from initiation to necrosis could be classified into four characteristic stages and 
that tumor vascular morphology drastically changed according to tumor growth.

An effective drug accumulation in tumors can lead to a significant therapeutic response for the outcome of treatment. ${ }^{10,11}$ Strategies that can further increase drug availability and therapeutic responses in tumor inhibition are needed. Ultrasound (US) sonication with microbubbles (MBs) could noninvasively enhance the transport of therapeutic agents to targeted tissues. ${ }^{12,13}$ With US sonication right after an injection of MBs, the MBs in the vasculature within the acoustic beam are interacting with the ultrasound waves. The interaction of ultrasound waves with MBs may cause oscillation and cavitation of the bubbles and lead to the rupture of vascular walls. ${ }^{14-16}$ For overcoming the difficulties of drug delivery posed by vascular walls, US sonication in the presence of MBs can produce ruptured openings to increase vascular permeability of sonicated tissues and permit a specific and effective cellular uptake. ${ }^{17-20}$ Studies have recently demonstrated that ultrasoundmediated chemotherapeutics with MBs showed promising potential for animal tumor treatment. ${ }^{19,20}$ The therapeutic drug concentration increased specifically in the sonicated region and significantly suppressed the tumor growth.

Pegylated liposomal doxorubicin (PLD) is able to avoid the recognition of the reticuloendothelial system and possesses long-circulating properties to improve the delivery of free drug. ${ }^{21-23}$ The average size of PLD is approximately $100 \mathrm{~nm}$, which is small enough for the PLD to extravasate through the leaky vasculature to achieve passive accumulation in tumor tissues. Previously, we have shown that focused US (FUS) sonication with MBs can significantly enhance the delivery of nanoparticles into tumor tissues. ${ }^{15,16}$ The results indicated that an injection of MBs followed by pulsed-FUS sonication is promising for nanodrug delivery in the sonicated tumor tissues. There have been no reports relating to the influence of the changes of vascular permeability through ruptured openings on the tumor treatment at different tumor growth stages. In the present study, we employ a mouse-ear tumor model to investigate the influences of US sonication in the presence of MBs on the therapeutic responses of early and medium-stage tumors. Different PLD dosages were also considered, and histological examination and blood perfusion measurement were used to study the tumors' physiological changes due to US sonication with MBs.

\section{Materials and methods Reagents}

The PLD (Doxil) was obtained from Alza (Bedford, MA). The US contrast agent (UCA) (SonoVue) used as MBs was purchased from Bracco (Amsterdam, Netherlands). Other chemicals, if not specified, were reagent grade from Aldrich-Sigma (St, Louis, MO).

\section{Cell culture and animal model}

Mouse colorectal adenocarcinoma CT-26 cells were received from American Type Culture Collection (ATCC, MD). The CT-26 cells were cultured in the Roswell Park Memorial Institute medium supplemented with $10 \%$ fetal bovine serum in a $37^{\circ} \mathrm{C}, 5 \% \mathrm{CO}_{2}$ incubator for 24 hours with complete cell attachment. Cell number and viability were counted by using a hemocytometer with trypan blue exclusion test.

Male, 6-week-old BALB/c mice weighing 20-25 g were used in this study. To produce ear tumors for experiments, CT- 26 cells $\left(10^{6}\right.$ cells $\left./ 50 \mu \mathrm{L}\right)$ were inoculated subcutaneously into both sides of the mouse ears. Before the experiments, tumor size was selected according to tumor volume (TV) by the measurement of length $(L)$, width $(W)$, and height $(H)$ with a caliper and using the formula $T V=\pi / 6 L \times W \times H \cdot{ }^{24}$ All tumors were measured three times per week. Seven or 10 days after tumor cell inoculation, mice bearing tumors with an initial size of about $15 \mathrm{~mm}^{3}$ (early stage) or $50 \mathrm{~mm}^{3}$ (medium stage) were selected for the experiments. The mice were anesthetized with a combined solution of ketamine $(30 \mathrm{mg} / \mathrm{kg})$ and acepromazine $(0.75 \mathrm{mg} / \mathrm{kg})$ by intraperitoneal injection. The experiment procedure met the criteria outlined by the Institution of Animal Care and Use Committee of National Taiwan University, and the mice were handled according to the guidelines in The Handbook of Laboratory Animal Center, National Taiwan University. All experiments were designed to minimize the animals' suffering.

\section{Ultrasound sonication system}

The sonication was conducted either with a $1.0-\mathrm{MHz}$ US transducer (Sonitron 2000; Rich Mar, Inola, OK; output power intensity $2 \mathrm{~W} / \mathrm{cm}^{2}$, duty cycle $50 \%$, pulse length $10 \mathrm{~ms}$, pulse-repetition frequency $50 \mathrm{~Hz}$, and sonication duration 60 seconds) for early stage tumors or with a 1.0MHz FUS transducer (A392S; Panametrics, Waltham, MA; diameter $38 \mathrm{~mm}$, curvature radius $63.5 \mathrm{~mm}$ ) for mediumstage tumors, which was driven by a power amplifier (150A250A; Amplifier Research, Souderton, PA) connected to both a function generator (33220A; Agilent, Palo Alto, CA) and a power meter/sensor module (4421; Bird, Cleveland, $\mathrm{OH}$ ). A removable cone (inner diameters for top and bottom 12 and $38 \mathrm{~mm}$, respectively; cone length $58 \mathrm{~mm}$ ) filled with distilled and degassed water was mounted on the bottom of the FUS transducer. The tip of the cone was 
firmly covered with a polyurethane membrane to ensure no air bubbles in the water, and the cone was used to guide the acoustic beam to the tumor region. The center of the focal zone was about $5.3 \mathrm{~mm}$ away from the cone tip. The acoustic pressure at the focal point was $0.6 \mathrm{MPa}$, measured by a needle hydrophone (HPM1/1; Precision Acoustics, Dorchester, UK), and the parameters of FUS sonication used were 60 -second sonication duration, a 10 -ms pulse length, a $50 \%$ duty cycle, and a $50-\mathrm{Hz}$ pulse-repetition frequency. A 3-mm-thick acoustic transmission gel (Pharmaceutical Innovations, Newark, NJ) was mantled to the skin over each tumor, and then the cone tip was immersed in the gel and targeted on the tumor. The cone with US transducer was then circularly scanned above the tumor and sonicated, as shown in Figure 1.

\section{Experimental designs}

We conducted the experiments to investigate the influence of US sonication with MBs on the tumor growth in mouse-ear tumors treated with anticancer nanodrug. In this study, a US contrast agent (SonoVue; phospholipid-coated MBs mean diameter $2.5 \mu \mathrm{m}$ and concentration $2-5 \times 10^{8}$ bubbles $/ \mathrm{mL}$ ) was used as MBs. If not specified, the injected dose of MBs was $100 \mu \mathrm{L} / \mathrm{kg}$.

\section{Growth response for early stage tumors treated with $10 \mathrm{mg} / \mathrm{kg}$ of PLD}

To study the effect of US sonication with MBs on the therapeutic response of early stage tumors, we selected a small US probe ( $3 \mathrm{~mm}$ diameter at the tip) and an initial size of treated tumors of about $15 \mathrm{~mm}^{3}$ (7 days after inoculation). Experimental groups included the control $(n=3)$, PLD (PLD injection only, $\mathrm{n}=6$ ), PLD + MBs + US (PLD injection, followed by MBs injection and then US sonication, $n=6)$, US (US sonication only, $\mathrm{n}=3$ ), and MBs + US (MBs injection, followed by US sonication, $\mathrm{n}=3$ ). We injected $10 \mathrm{mg} / \mathrm{kg}$ of PLD into the tail veins of the tumor-bearing mice for both PLD and PLD + MBs + US groups, and the other two groups were treated with either MBs + US or US alone, respectively, on days 7,14 , and 21 after tumor inoculation.

\section{Growth response for medium-stage tumors treated with $6 \mathrm{mg} / \mathrm{kg}$ of PLD}

To study the effect of US sonication with MBs on the therapeutic response of medium-stage tumors, we used an FUS transducer and an initial size of treated tumor of about $50 \mathrm{~mm}^{3}$ (10 days after inoculation). The mice were divided into five groups: the control $(n=6), \operatorname{PLD}(n=5)$, the PLD + MBs + US $(n=5), U S(n=3)$, and MBs + US $(n=3)$.

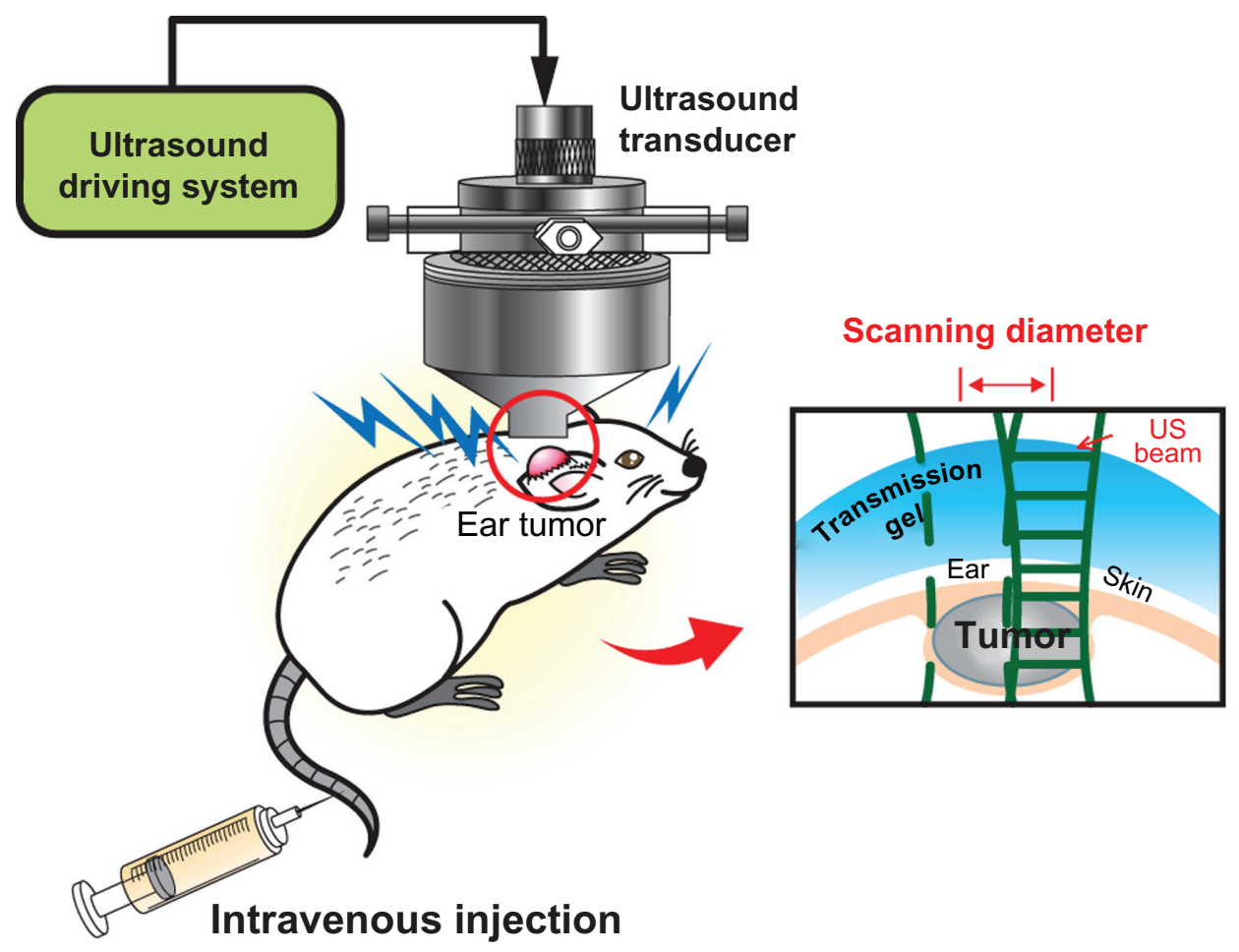

Figure I Experimental arrangement. The acoustic beam from the ultrasound transducer to sonicate mouse ear tumors was guided by a cone filled with degassed water. A 3-mm-thick ultrasound transmission gel was mantled over the ear skin and the acoustic beam was targeted on the tumor. The ultrasound beam was circularly scanned during sonication. 
Treatments were executed with an injection of $6 \mathrm{mg} / \mathrm{kg}$ PLD for both the PLD and PLD + MBs + US groups, and the other two groups were treated with either MBs + US or US alone, respectively, on days 10 and 17 after tumor inoculation.

\section{Growth response for medium-stage tumors treated} with $4 \mathrm{mg} / \mathrm{kg}$ of PLD

To further investigate the effect of MBs + US on the therapeutic response, a lower dose $(4 \mathrm{mg} / \mathrm{kg})$ of PLD was used to treat tumors with an initial size of about $50 \mathrm{~mm}^{3}$ for both the PLD and PLD + MBs + US groups. The treatments were executed on days 10 and 17 after tumor inoculation.

\section{Measurements of tumor blood perfusion with a Laser Doppler}

Red blood cell perfusion was measured using a laser Doppler (OxyLab LDF; Oxford Optronics, Oxford, UK). The mice bearing ear tumors were divided into three groups: the control group $(n=3)$, the PLD group $(n=3)$, and the PLD + MBs + US group $(n=5)$. Blood perfusion of the ear tumors was assessed just before both the first treatment (on day 10 after tumor inoculation) and the second treatment (on day 17). For each tumor, red blood cell perfusion was obtained from one central and two peripheral locations of the tumor using the Miniature Surface Probe (MSP310XP [diameter $1 \mathrm{~mm}$ ]; AD Instruments, Bella Vista, NSW, Australia) with a self-adhesive disc. Estimates of perfusion were obtained at a frequency of $5 \mathrm{~Hz}$ over a period of 60 seconds. The measurements for each sample were repeated three times, and the mean value in blood perfusion units was calculated. The data were expressed as relative blood perfusion units with percentage values.

\section{Microscopic system and histological morphology}

Tumor development on both ears was observed every day after tumor inoculation, and the mice were anesthetized and photomicrographed every other day for both ears under transillumination using a Leica MZ16FA stereomicroscope (Leica Microsystems, Wetzlar, Germany).

The mice were killed at the end of the experiment, and were then infused with $0.9 \%$ saline via the left ventricle. After infusion, tumor tissues were harvested from both ears, and the tissues were immediately frozen in liquid nitrogen and kept at $-80^{\circ} \mathrm{C}$ for later histological staining.

Mouse tumor tissues were moved from the $-80^{\circ} \mathrm{C}$ freezer to $\mathrm{a}-20^{\circ} \mathrm{C}$ Leica CM3050 S Cryostat (Meyer, TX) and sectioned. The tissues were embedded into a Tissue-Tek
OCT compound (Sakura Finetek, Torrance, CA) and were sectioned into 5 - $\mu \mathrm{m}$-thick slices on SuperFrost glass slides (Thermo Fisher Scientific, Braunschweig, Germany). Tissue slices were stained with hematoxylin and eosin (H\&E) for histological analysis and evaluated using a Leica DMIRB microscope (Leica Microsystems) with image acquisition software to examine the slices of H\&E staining.

\section{Statistical analysis}

Statistical analysis of variance was used to evaluate the data, and a nonparametric Mann-Whitney $U$ test was used to determine the level of significance of differences in sample means with the Statistical Package for the Social Sciences version 16.0 software (SPSS, Chicago, IL). Values of $P<0.05$ and $P<0.01$ were considered significant.

\section{Results \\ Influence of US sonication with MBs on tumor growth for early stage tumors treated with anticancer nanodrug}

To investigate the therapeutic effects of US sonication with MBs on early stage tumors treated with anticancer nanodrug, we injected $10 \mathrm{mg} / \mathrm{kg}$ of PLD through the tail vein of the mice with an initial size of treated ear tumor of about $15 \mathrm{~mm}^{3}$ for both PLD and PLD + MBs + US groups, whereas the other two groups received US sonication with or without MBs injection. Figure 2 shows the tumor growth response during a sequence of treatments on days 7, 14, and 21 after tumor inoculation. The results show that the tumor growth is significantly inhibited in the PLD, PLD + MBs + US, and MBs + US groups, as compared with the control group $(P<0.05, P<0.01)$. The results display that US sonication with MBs after an injection of $10 \mathrm{mg} / \mathrm{kg}$ PLD can further and earlier inhibit the tumor growth, as compared with PLD alone. It is also worth noting that tumor growth appears biphasic: the size increases after the first treatment and then decreases, for both PLD and PLD + MBs + US groups. Meanwhile, tumor growth for the MBs + US group was hindered, even though there was no PLD injection.

\section{Influences of FUS sonication with MBs on tumor growth and blood perfusion for medium-stage tumors treated with anticancer nanodrug}

To investigate the therapeutic effects of FUS sonication with MBs on medium-stage tumors treated with anticancer nanodrug, we injected $6 \mathrm{mg} / \mathrm{kg}$ of PLD into the mice with an 


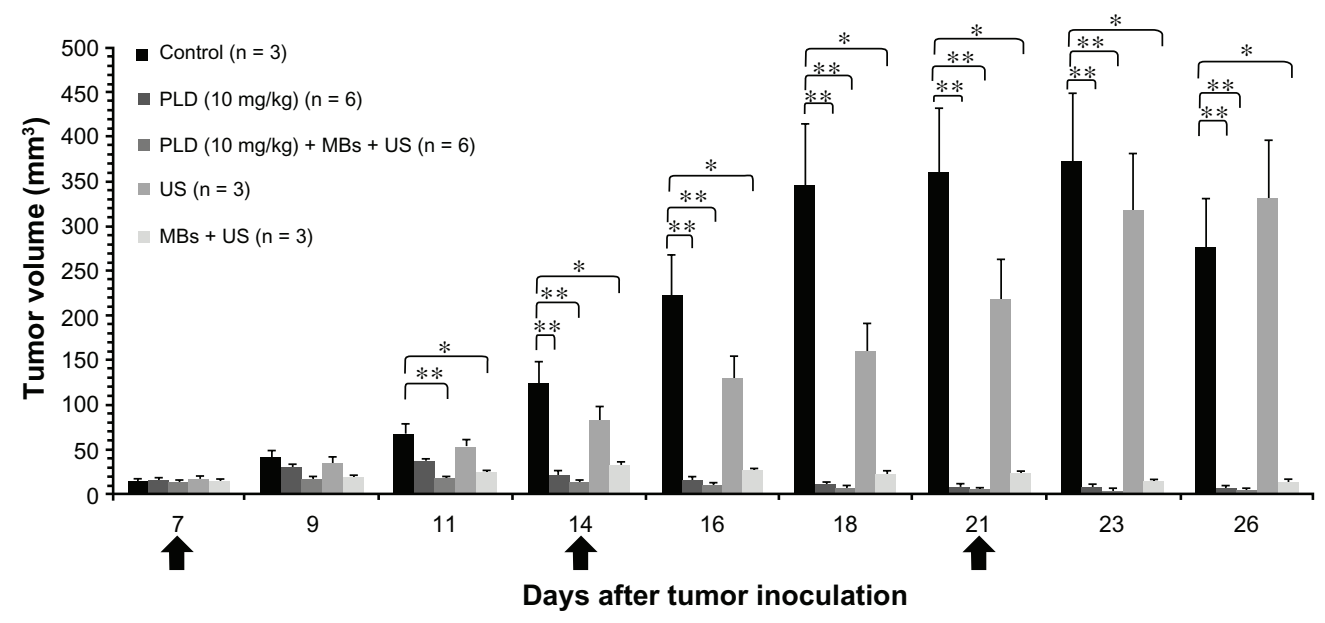

Figure 2 Effects of ultrasound (US) sonication with microbubbles (MBs) on tumor growth for early stage tumors treated with $10 \mathrm{mg} / \mathrm{kg}$ of anticancer nanodrug. The arrows indicate the schedule for treatments. The figure shows the tumor growth responses for an initial size of treated tumors of about 15 mm ${ }^{3}$ with different conditions: control, PLD alone, PLD + MBs + US, US alone, and MBs + US.

Notes: $* P<0.05 ; * * P<0.0$ I (Mann-Whitney $U$ test); for each group, mean \pm standard deviation.

Abbreviations: US, ultrasound; MBs, microbubbles; PLD, pegylated liposomal doxorubicin.

initial size of treated ear tumor of about $50 \mathrm{~mm}^{3}$ for both PLD and PLD + MBs + US groups, whereas the other two groups received FUS sonication with or without MBs injection. Figure 3A shows the tumor growth responses for treatments on days 10 and 17 after tumor inoculation. This figure displays that the tumor growth was effectively inhibited after the first treatment for the PLD group $(P<0.05$, as compared with the control group), and the tumor growth was further inhibited by FUS sonication with MBs $(P<0.01$ between the PLD + MBs + US and control groups). Meanwhile, there was no significant difference among the control, US, and MBs + US groups.

Photomicrograph was used to examine the tumor growth for the control, PLD, and PLD + MBs + US groups on days 10,16 , and 26 after tumor inoculation. Figure 3B, photomicrograph observation for the tumors, shows that the inhibition of tumor growth was more effective in the PLD + MBs + US group than the PLD group, and the tumor almost disappeared for the former.

Laser Doppler was used to examine the response of microvascular blood perfusion for the tumors after treatment. We measured the tumor blood perfusion for the control, PLD, and PLD + MBs + US groups just before both the first treatment (day 10 after tumor inoculation) and the second treatment (day 17 after inoculation). Figure 3C shows that blood perfusion significantly decreased 7 days after the first treatment for the PLD group $(P<0.05)$, and it was further decreased by FUS sonication with MBs (the PLD + MBs + US group $)(P<0.01)$.

\section{Histological examinations for the influence of FUS sonication with MBs on tumor tissues}

Staining with H\&E was used to examine the change of histology induced in tumor tissues for the PLD, PLD + MBs + US, and MBs + US groups. The H\&E staining of tumor tissues for these three groups is shown in Figure 4, depicting the treatment results of Figure 3. Hemorrhagic damage occurred rarely in the PLD and MBs + US groups, whereas for the PLD + MBs + US group, FUS sonication with MBs resulted in local suppression of neutrophil influx, interstitial edema, congestion, and disruption of tissue architecture.

\section{Influence of FUS sonication with MBs on tumor growth for medium-stage tumors with a low dose of anticancer nanodrug}

To further study the effect of FUS sonication with MBs on the therapeutic response of anticancer nanodrug for medium-stage tumors, we injected a low dose $(4 \mathrm{mg} / \mathrm{kg})$ of PLD into the mice with an initial treated tumor of size about $50 \mathrm{~mm}^{3}$ for both PLD and PLD + MBs + US groups. Figure 5 shows the tumor growth response with treatments on days 10 and 17 after inoculation. The results show that the tumor growth was significantly different between the PLD and PLD + MBs + US groups. There was no hindrance to the tumor growth for the PLD group, while the application of FUS sonication with MBs after the injection of PLD was able to significantly inhibit tumor growth. 


\section{A}

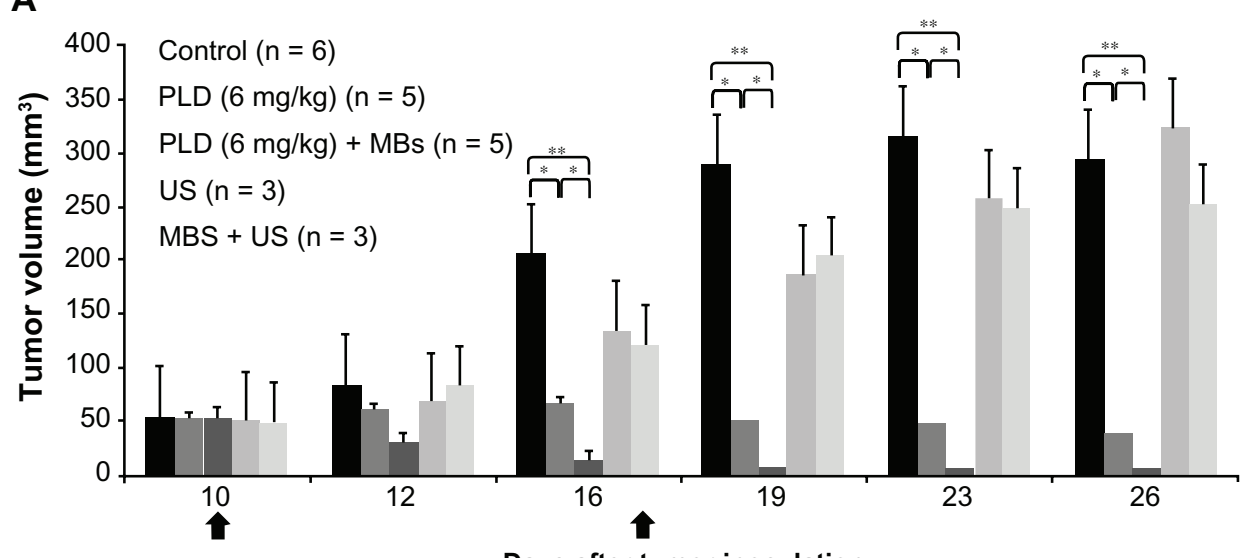

Days after tumor inoculation

B
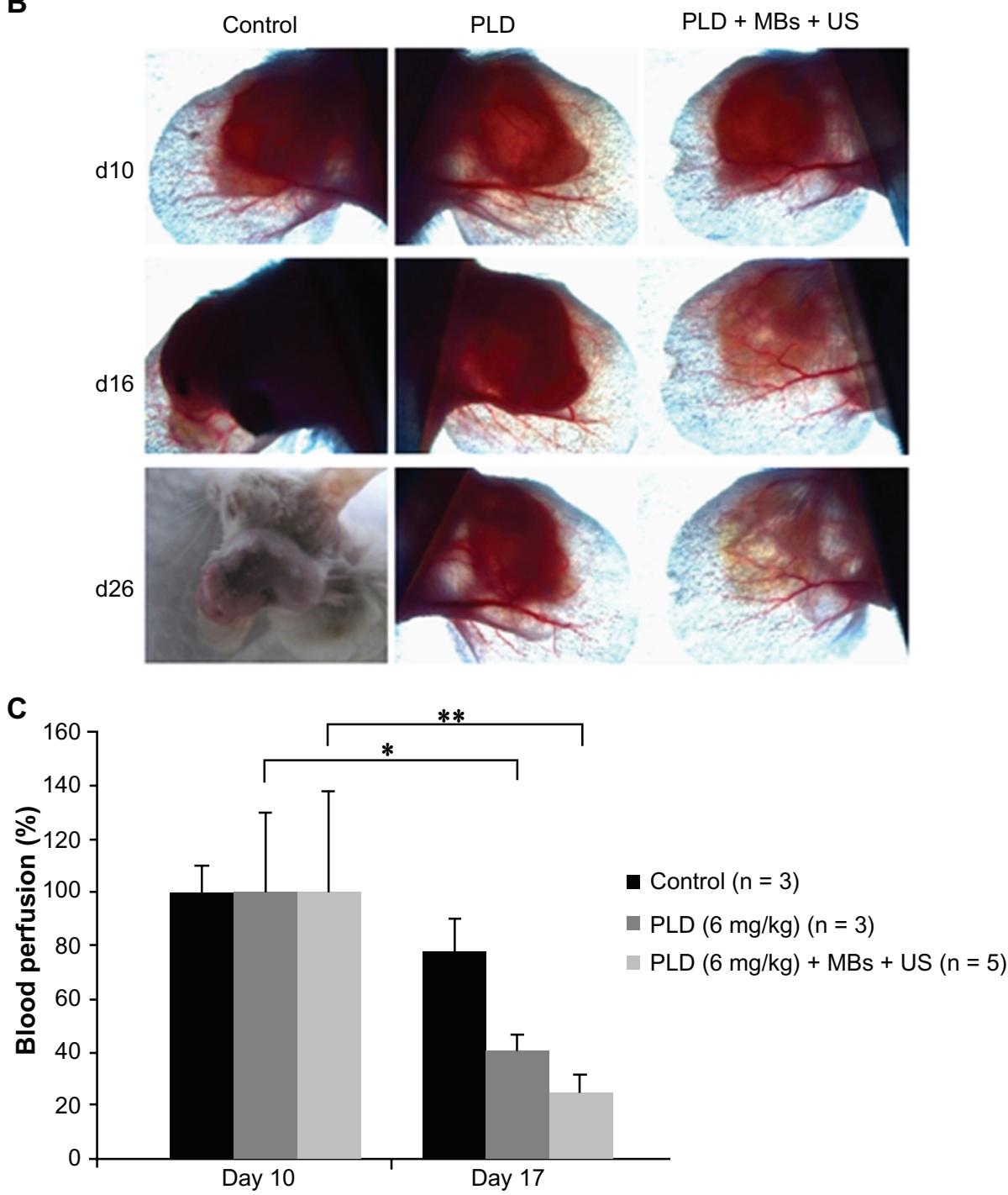

Days after tumor inoculation

Figure 3 Effects of focused US sonication with MBs on tumor growth for medium-stage tumors treated with 6 mg/kg. (A) Tumor growth responses for an initial size of treated tumors of about $50 \mathrm{~mm}^{3}$ with different conditions: control, PLD alone, PLD + MBs + US, US alone, and MBs + US. The arrows indicate the schedule for treatments. (B) Photomicrographs of mouse-ear tumors (control, PLD alone, and PLD + MBs + US) on days 10, 16, and 26 after tumor inoculation (magnification: 10X). (C) Blood perfusion measurements of tumors before treatment on days 10 and 17, for the control, PLD, and PLD + MBs + US groups.

Notes: Mean $\pm S D ; * P<0.05$, **P $<0.0$ l (Mann-Whitney $U$ test)

Abbreviations: US, ultrasound; MBs, microbubbles; PLD, pegylated liposomal doxorubicin. 


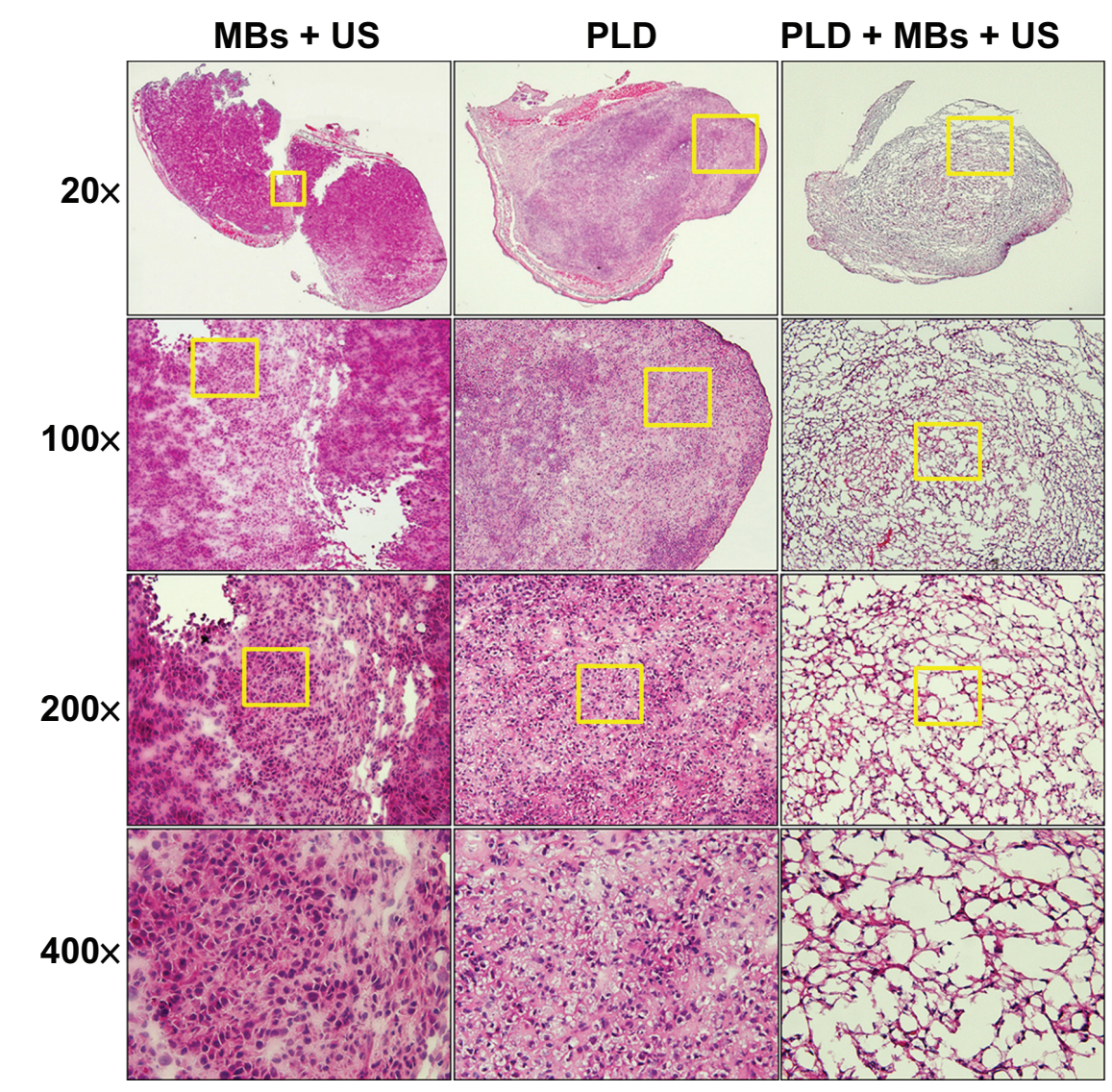

Figure $4 \mathrm{H} \& \mathrm{E}$ staining of tissue sections for the ear tumors after a sequence of treatments with MBs + US, PLD alone, and PLD + MBs + US. Notes: Magnifications 20×, 100×, 200x, and 400× (yellow square).

Abbreviations: H\&E, hematoxylin and eosin; MBs, microbubbles; US, ultrasound; PLD, pegylated liposomal doxorubicin.

\section{Discussion}

In this study, we investigated the synergistic effect of US sonication with MBs on early and medium staged tumors located on mouse ears treated with anticancer nanodrug. The results showed that: (1) US sonication in the presence of MBs was always able to produce a further hindrance to tumor growth for both early and medium-stage tumors; (2) the dosage of anticancer nanodrug was one of the critical factors for the tumor growth inhibition: administration of $6 \mathrm{mg} / \mathrm{kg}$ PLD alone was able to inhibit the growth of medium-stage tumors, while this did not occur for injection of $4 \mathrm{mg} / \mathrm{kg}$ PLD alone; (3) with the application of MBs + US after nanodrug administration, a low dose of PLD (4 mg/ $\mathrm{kg}$ ) was able to inhibit the growth of medium-stage tumors; (4) for early stage tumors after the first treatment with a high dose of nanodrug alone (PLD $10 \mathrm{mg} / \mathrm{kg}$ ), the tumor size still increased for several days and then decreased (biphasic pattern); (5) the use of MBs + US alone was able to hinder the tumor growth of early stage tumors, but for medium-stage tumors MBs + US alone could not inhibit tumor growth, and an injection of PLD was required for effective hindrance.
The experimental results indicated that the use of MBs + US can significantly affect the growth response of early stage tumors (Figure 2) and the application of MBs + US after PLD administration can enhance the anticancer treatment of PLD to further inhibit tumor growth for both early and medium-stage tumors (Figures 2, 3, and 5).

When US sonicates in the tumors right after an injection of MBs, the interaction of the US beam with MBs in blood vessels results in the bubbles' oscillation, collapse, and even violent cavitation. These phenomena of MBs may disrupt blood vessel walls and produce vascular pores or even vascular rupture, which may be able to hinder the growth of early stage tumors (Figure 2) and improve the transport of nanodrug into tumor tissues to further inhibit tumor growth when PLD is injected before the use of US + MBs for both early and medium-stage tumors (Figures 2, 3, and 5). Meanwhile, the temperature rise in the sonicated tumor tissue during US sonication in the presence of MBs may have some thermal effects on the tumor to increase the blood flow for raising the delivery of nanodrug into tumor tissues. 


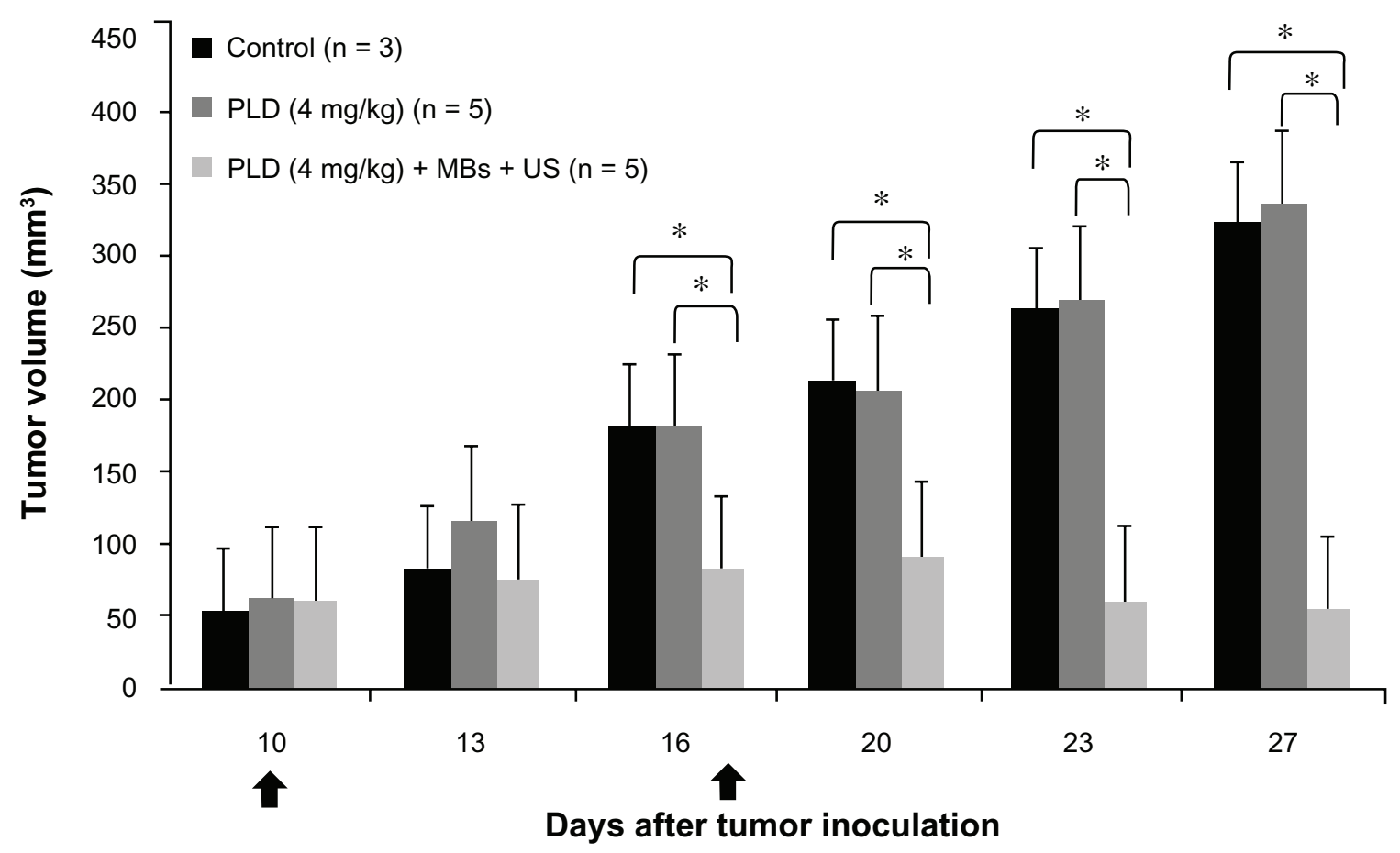

Figure 5 Tumor growth response of medium-staged tumors treated with $4 \mathrm{mg} / \mathrm{kg}$ of PLD with and without MBs + FUS. The arrows indicate the schedule for treatments. Notes: Mean \pm SD; $* P<0.05$ (Mann-Whitney $U$ test).

Abbreviations: PLD, pegylated liposomal doxorubicin; MBs, microbubbles; FUS, focused ultrasound.

The vascular transport of anticancer nanodrug into tumor tissues is related to both the stage of tumor growth ${ }^{9,25}$ and the use of MBs + US. For early stage tumors, the response of tumor growth displays a biphasic pattern (tumor size increases for several days and then decreases) after the first treatment with a high dose of PLD $(10 \mathrm{mg} / \mathrm{kg})$ alone. For early stage tumors, their vascular integrity is still rather intact for PLD and hence it impedes the transport of nanodrug into the tumor tissue, ${ }^{21,25}$ while the vascular permeability for PLD increases as the tumor enters the medium stage. This may be able to explain the phenomenon that early stage tumors still grew for several days after injection of a high dose of PLD $(10 \mathrm{mg} / \mathrm{kg})$, while medium-stage tumors were inhibited after a $6 \mathrm{mg} / \mathrm{kg}$ dose of PLD. It is also worth noting that the growth of early stage tumors after the first treatment was significantly hindered for the MBs + US group (Figure 2). This indicates that the bubbles' oscillation, collapse, and violent cavitation in microvessels during US sonication disrupt microvessels sufficiently to affect the growth of early stage tumors. Previous studies displayed that early stage tumors have a much higher microvascular density, ${ }^{9,25}$ and a prominent antivascular effect on tumors was produced after US sonication with MBs. ${ }^{26,27}$ Histology demonstrated disruption of vascular walls and tumor cell death in areas of vascular congestion and thrombosis. ${ }^{28}$ For early stage tumors, their microvasculature is denser but fragile, and hence more thorough damage may appear to suppress tumor growth after US sonication with MBs. The damage level of blood vessels to hinder tumor growth due to US sonication depends on tumor growth stage, quantity of injected MBs, and sonication conditions, which may be able to explain the hindrance difference between early and medium-stage tumors for the MBs + US groups in Figures 2 and 3.

The transport of nanodrug and its concentration distribution in tumor tissues are related to (1) convection, in which fluid flows through the vascular walls into tumor tissues and from tumor tissues to the adjacent normal tissues, (2) diffusion, which is determined by concentration difference of nanodrug between intravascular and extravascular regions, and (3) injected dosage of nanodrug. Hence, vascular density, vascular permeability, and interstitial diffusivity of PLD nanodrug, interstitial pressure in tumors, and injected dosage are crucial factors affecting the anticancer treatment. Early stage tumors possess high vascular density, but with relatively intact vascular walls hampering the transport of PLD into tumor tissues. Hence the use of MBs + US to disrupt vascular integrity can effectively induce the transport of nanodrug into interstitial tumor tissues to hinder tumor growth. This may be able to explain the reason for the hindrance to tumor growth for the PLD + MBs + US group $(P<0.01$ compared with the 
control group) appearing significantly earlier than the PLD group, as shown in Figure 2. For medium-stage tumors (about $50 \mathrm{~mm}^{3}$ ), they have already developed pores on vascular walls sufficiently large for the transport of PLD nanodrug to the extravascular region, while their vascular density is relatively less. It means that there is a longer distance for drug to travel to reach a therapeutic level in some tumor region. An administration of $6 \mathrm{mg} / \mathrm{kg}$ PLD is able to inhibit tumor growth, while an injection of $4 \mathrm{mg} / \mathrm{kg}$ PLD cannot result in a sufficiently high dose of drug in some tumor regions, due to the long-distance transport of drug, to hinder medium-stage tumor growth. FUS sonication with MBs is able to disrupt blood vessels to enable greater transport of PLD (Figure 4), making it possible to inhibit tumor growth with a dose as low as $4 \mathrm{mg} / \mathrm{kg}$ (Figure 5). This suggests that the use of MBs + US may reduce the dosage of PLD down to $4 \mathrm{mg} / \mathrm{kg}$, which may avoid toxicities while maintaining antitumor efficacy. In addition to vascular density and permeability, other tumor microenvironment conditions also need to be considered, such as high interstitial fluid pressure, hypoxia, and low $\mathrm{pH}$, which may hinder drug delivery and treatment effectiveness for a low dose of PLD to inhibit the growth of medium-stage tumors.

Tumor perfusion measured by laser Doppler showed that the reduction of blood perfusion was statistically significant for both the PLD and PLD + MBs + US groups $(P<0.05$, $P<0.01)$ after one treatment, as shown in Figure 3C. As compared with Figure $3 \mathrm{~A}$, the tumor growth was significantly inhibited for the PLD group, and the tumors even shrank significantly for the PLD + MBs + US group. This suggests that vasculature was effectively damaged by PLD treatment and further damaged with the application of MBs + US, while the reduction of blood perfusion in the control group can be regarded as due to the significant growth of tumor size. During US sonication in the presence of MBs, the oscillation, collapse, and cavitation of MBs in the acoustic beam produced vascular pores and disruption of vessel wall to increase vascular permeability significantly in the sonicated areas. This may have further led to the formation of smaller bubbles, which interacted with the US beam and caused the cellular bioeffects. The predominant acute effects of US sonication might have induced dilation of tumor vessels and hemorrhage, so that there would not have been the normal structural support for the capillaries. As the vessel wall was no longer functioning well, it was incapable of counteracting the intravascular pressure, and then the capillaries dilated and became leaky, even cascading to edema. Figure 4 shows the histological results, in which the peritumor edema was a characteristic finding in the tumor morphology. This implies that the mild intercellular fluid across the entire tumor with nanodrug solution might further induce tumor damage for the PLD + MBs + US group, while MBs + US alone enhanced the flow perfusion without cellular damage. Therefore, nanodrug delivery enhancement with MBs + US was correlated with structural changes created in the tissue.

In this study, a significant hindrance to tumor growth was achieved using MBs + US to disrupt tumor blood vessels, while targeted delivery of nanodrug into tumor tissue was enhanced. However, the associated toxicities may appear in other tissue when the high-pressure region of the US beam is scanned through those tissues, and it is likely to cause some vascular damage and chemotherapeutic effects on sonicated nontumor tissue. Furthermore, the disruption of blood vessels in the sonicated tumor can enhance the delivery of nanodrug into tumor tissues, while it may have a double-edged-sword effect in facilitating the intravasation of tumor cells into circulation to increase metastasis. In future studies, it will be valuable to investigate the possibility of metastasis increase for the PLD + MBs + US group.

\section{Acknowledgments}

This work was supported by the National Health Research Institutes (No NHRI: ME-100-PP-13) and the National Science Council of Taiwan (No NSC: 99-2221-E-002-005-MY3).

\section{Disclosure}

The authors report no conflicts of interest in this work.

\section{References}

1. You S, Zuo L, Li W. Optimizing the time of Doxil injection to increase the drug retention in transplanted murine mammary tumors. Int J Nanomedicine. 2010;5:221-229.

2. Matsumura Y, Maeda H. A new concept for macromolecular therapeutics in cancer chemotherapy: mechanism of tumoritropic accumulation of proteins and the antitumor agent smancs. Cancer Res. 1986;46: 6387-9632.

3. Ferrari M. Cancer nanotechnology: opportunity and challenges. Nat Rev Cancer. 2005;5:161-171

4. Matsumura Y. Poly(amino acid) micelle nanocarriers in preclinical and clinical studies. Adv Drug Deliv Rev. 2008;60:899-914.

5. Senger DR, Galli SJ, Dvorak AM, Perruzzi CA, Harvey VS, Dvorak HF. Tumor cells secrete a vascular permeability factor that promotes accumulation of ascites fluid. Science. 1983;219:983-985.

6. Dvorak HF, Sioussat TM, Brown LF, et al. Distribution of vascular permeability factor (vascular endothelial growth factor) in tumors: concentration in tumor blood vessels. J Exp Med. 1991;174: 1275-1278

7. Ferrara N, Gerber HP, LeCouter J. The biology of VEGF and its receptors. Nat Med. 2003;9:669-676.

8. Jain RK. Barriers to drug delivery in solid tumors. Sci Am. 1994;271: $58-65$.

9. Yamaura H, Sato H. Quantitative studies on the developing vascular system of rat hepatoma. J Natl Cancer Inst. 1974;53:1229-1240. 
10. Silva AC, Oliveira TR, Mamani JB, et al. Application of hyperthermia induced by superparamagnetic iron oxide nanoparticles in glioma treatment. Int J Nanomedicine. 2011;6:591-603.

11. Maeda H. Tumor-selective delivery of macromolecular drugs via the EPR effect: background and future prospects. Bioconjugate Chem. 2010;21:797-802.

12. Hancock HA, Smith LH, Cuesta J, et al. Investigations into pulsed highintensity focused ultrasound-enhanced delivery: preliminary evidence for a novel mechanism. Ultrasound Med Biol. 2009;35:1722-1736.

13. Bohmer MR, Chlon CHT, Raju BI, et al. Focused ultrasound and microbubbles for enhanced extravasation. J Control Release. 2010;148: 18-24.

14. Zhao YZ, Lu CT, Zhou ZC, et al. Enhancing chemotherapeutic drug inhibition on tumor growth by ultrasound: an in vivo experiment. J Drug Target. 2011;19:154-160.

15. Lin CY, Huang YL, Li JR, Chang FH, Lin WL. Effects of focused ultrasound and microbubbles on the vascular permeability of nanoparticles delivered into mouse tumors. Ultrasound Med Biol. 2010;36:1460-1469.

16. Lin CY, Liu TM, Chen CY, et al. Quantitative and qualitative investigation into the impact of focused ultrasound with microbubbles on the triggered release of nanoparticles from vasculature in mouse tumors. J Control Release. 2010;146:291-298.

17. Rapoport N, Kennedy AM, Shea JE, et al. Controlled and targeted tumor chemotherapy by ultrasound-activated nanoemulsions/microbubbles. J Control Release. 2009;138:268-276.

18. Coussios CC, Farny CH, Haar GT, Roy RA. Role of acoustic cavitation in the delivery and monitoring of cancer treatment by high-intensity focused ultrasound (HIFU). Int J Hyperthermia. 2007;23:105-120.

19. Price R, Chappell J, Song J, Klibanov A. Nanoparticle delivery into biological tissues by ultrasonic microbubble destruction. Nanomedicine. 2006;2:269-312.
20. Frenkel V, Etherington A, Greene M, et al. Delivery of liposomal doxorubicin (Doxil) in a breast cancer tumor model: investigation of potential enhancement by pulsed-high intensity focused ultrasound exposure. Acad Radiol. 2006;4:469-479.

21. Hori K, Nishihara M, Shiraishi K, Yokoyama M. The combretastatin derivative (Cderiv), a vascular disrupting agent, enables polymeric nanomedicelles to accumulate in microtumors. J Pharm Sci. 2010;99: 2914-2925.

22. Huwyler J, Drewe J, Krahenbuhl S. Tumor targeting using liposomal antineoplastic drugs. Int J Nanomedicine. 2008;3:21-29.

23. Gabizon A, Shiota R, Papahadjopuulos D. Pharmacokinetics and tissue distribution of doxorubicin encapsulated in stable liposomes with long circulation times. J Natl Cancer Inst. 1989;81:1484-1488.

24. Al-Jamal WT, Al-Jamal KT, Tian B, et al. Lipid-quantum dot bilayer vesicles enhance tumor cell uptake and retention in vitro and in vivo. ACS Nano 2008;2:408-418.

25. Hori K, Nishihara M, Yokoyama M. Vital microscopic analysis of polymeric micelle extravasation from tumor vessels: macromolecular delivery according to tumor vascular growth stage. J Pharm Sci. 2010;99:549-562.

26. Bunte RM, Ansaloni S, Sehgal CM, Lee WM, Wood AK. Histopathological observations of the antivascular effects of physiotherapy ultrasound on a murine neoplasm. Ultrasound Med Biol. 2006;32:453-461.

27. Wood AK, Bunte RM, Cohen JD, et al. The antivascular action of physiotherapy ultrasound on a murine tumor: role of a microbubble contrast agent. Ultrasound Med Biol. 2007;33:1901-1910.

28. Wood AK, Ansaloni S, Ziemer LS, Lee WM, Feldman MD, Sehgal CM. The antivascular action of physiotherapy ultrasound on murine tumors. Ultrasound Med Biol. 2005;31:1403-1410.
International Journal of Nanomedicine

\section{Publish your work in this journal}

The International Journal of Nanomedicine is an international, peerreviewed journal focusing on the application of nanotechnology in diagnostics, therapeutics, and drug delivery systems throughout the biomedical field. This journal is indexed on PubMed Central, MedLine, CAS, SciSearch $\AA$, Current Contents ${ }^{\circledR} /$ Clinical Medicine,

\section{Dovepress}

Journal Citation Reports/Science Edition, EMBase, Scopus and the Elsevier Bibliographic databases. The manuscript management system is completely online and includes a very quick and fair peer-review system, which is all easy to use. Visit http://www.dovepress.com/ testimonials.php to read real quotes from published authors. 